\title{
SOLUTION OF HYPERGEOMETRIC INTEGRAL EQUATIONS INVOLVING GENERALISED FUNCTIONS
}

\author{
by ADAM C. MCBRIDE \\ (Received 10th December 1973)
}

\section{Introduction}

In a previous paper (9), we introduced the spaces $F_{p, \mu}$ of testing-functions and the corresponding spaces $F_{p, \mu}^{\prime}$ of generalised functions. For $1 \leqq p \leqq \infty$, $F_{p}\left(=F_{p, 0}\right)$ is the linear space of all complex-valued measurable functions $\phi$ defined on $(0, \infty)$ which are infinitely differentiable on $(0, \infty)$ and for which $x^{k} \frac{d^{k} \phi}{d x^{k}} \in L^{p}(0, \infty)$ for each $k=0,1,2, \ldots$ In symbols,

$$
F_{p}=\left\{\phi \in C^{\infty}(0, \infty): x^{k} \frac{d^{k} \phi}{d x^{k}} \in L^{p}(0, \infty) \text { for } k=0,1,2, \ldots\right\}
$$

$F_{p}$ is equipped with the topology generated by the semi-norms $\left\{\gamma_{k}^{p}\right\}_{k=0}^{\infty}$ where, for $\phi \in F_{p}$,

$$
\gamma_{k}^{p}(\phi)=\left\|x^{k} \frac{d^{k} \phi}{d x^{k}}\right\|_{p}(k=0,1,2, \ldots)
$$

(and \|\|$_{p}$ denotes the usual $L^{p}(0, \infty)$ norm). Then, for any complex number $\mu$, and $\mathrm{l} \leqq p \leqq \infty$, we define $F_{p, \mu}$ by

$$
F_{p, \mu}=\left\{\phi: x^{-\mu} \phi(x) \in F_{p}\right\} .
$$

$F_{p, \mu}$ is equipped with the topology generated by the semi-norms $\left\{\gamma_{k}^{p, \mu}\right\}_{k}^{\infty}=0$ where, for $\phi \in F_{p, \mu}$,

$$
\gamma_{k}^{p, \mu}(\phi)=\gamma_{k}^{p}\left(x^{-\mu} \phi\right) \quad(k=0,1,2, \ldots)
$$

with $\gamma_{k}^{p}$ as in (1.2). Finally, $F_{p, \mu}^{\prime}$ is the linear space of continuous linear functionals on $F_{p, \mu}$; it is equipped with the topology of weak (or pointwise) convergence.

The spaces $F_{p, \mu}$ and $F_{p, \mu}^{\prime}$ are amenable to the study of various operators of fractional integration and, in (9), we investigated the mapping properties of $I_{x^{m}}^{z}$ and $K_{x^{m}}^{\alpha}$, the Riemann-Liouville and Weyl fractional integrals respectively, as well as the Erdélyi-Kober operators $I_{x^{m}}^{\eta, \alpha}$ and $K_{x^{m}}^{\eta, \alpha}$. We also gave a simple application to differential operators. In this paper, we are going to turn our attention to integral operators.

We shall be concerned with four operators $H_{i}(a, b ; c ; m)(i=1,2,3,4)$

$$
\text { E.M.S.-19/3-S }
$$


typical of which is $H_{1}(a, b ; c ; m)$, defined for $\operatorname{Re} c>0$ and suitable functions $\phi$ by

$H_{1}(a, b ; c ; m) \phi(x)=\int_{0}^{x} \frac{\left(x^{m}-t^{m}\right)^{c-1}}{\Gamma(c)} F\left(a, b ; c ; 1-\frac{x^{m}}{t^{m}}\right) m t^{m-1} \phi(t) d t$.

Here $m>0$ is real, $a, b$ and $c$ are complex and $F(a, b ; c ; z) \equiv{ }_{2} F_{1}(a, b ; c ; z)$ is the Gauss hypergeometric function. For $m=1$, the operators $H_{i}(a, b ; c ; m)$ have been discussed at length for classical functions by Love in (5) and (6). Love's results unified the work of many authors who had earlier treated particular cases; see (5) and (6) for references. Here we work with generalised functions in $F_{p, \mu}^{\prime}$ rather than classical functions. With tools such as analytic continuation available, it is not surprising that the restrictions on the parameters involved are not so numerous as in Love's work. On the other hand, we end up with a generalised solution which may or may not correspond to a classical solution. However, we shall give one result to indicate how, in a particular case, we can recover a classical solution.

In Section 2, we gather together a few facts about $F(a, b ; c ; z)$ which we require in the sequel. In Section 3 , we develop the properties of $H_{1}(a, b ; c ; m)$ on $F_{p, \mu}$ by establishing a connection with the operators $I_{x m}^{\eta, \alpha}$. Analytic continuation and results in (9) enable us to extend the definition of $H_{1}(a, b ; c ; m)$ to values of $c$ with $\operatorname{Re} c \leqq 0$ (although in this case we will no longer be able to use the integral representation (1.5)). In Section 4, we introduce the other three operators $H_{i}(a, b ; c ; m)(i=2,3,4)$ on $F_{p \mu}$ and obtain connections between them. $H_{3}(a, b ; c ; m)$ and $H_{4}(a, b ; c ; m)$ are the adjoints of $H_{2}(a, b ; c ; m)$ and $H_{1}(a, b ; c ; m)$ respectively and moreover we find that

$$
\left[H_{2}(a, b ; c ; m)\right]^{-1}=H_{1}(-a,-b ;-c ; m)
$$

a fact which does not emerge clearly in (5).

The use of adjoint operators enables us to define the operators

$$
H_{i}(a, b ; c ; m) \quad(i=1,2,3,4)
$$

on $F_{p, \mu}^{\prime}$ and in Section 5 we obtain their mapping properties as well as giving formulae for the solution $f$ of

$$
H_{i}(a, b ; c ; m) f=g,
$$

where $g$ is a given generalised function. Finally, in Section 6, we compare and contrast our results with those of Love and also discuss the problem of finding classical solutions.

In the course of our travels, we establish on $F_{p, \mu}$ and $F_{p, \mu}^{\prime}$ the second index laws for the operators $I_{x^{m}}^{\alpha}$ and $K_{x^{m}}^{\alpha}$; if $\alpha+\beta+\gamma=0$, then

$$
\begin{aligned}
x^{m \alpha} I_{x^{m}}^{\beta} x^{m v} f & =I_{x^{m}}^{-\gamma} x^{-m \beta} I_{x^{m}}^{-\alpha} f \\
x^{m y} K_{x^{m}}^{\beta} x^{m x} f & =K_{x^{m}}^{-\alpha} x^{-m \beta} K_{x^{m}}^{-\gamma} f
\end{aligned}
$$

are valid under appropriate conditions. These arise naturally in the discussions; they have been discussed for classical functions by Love (7) and for a class of generalised functions by Erdélyi (1). 
Throughout, we shall use the notation and terminology of (9). In particular, we shall assume that $m>0$, that $1 \leqq p \leqq \infty$ (unless the contrary is stated) and that $p, q$ are connected by $1 / p+1 / q=1$.

\section{2}

We shall denote Gauss' hypergeometric function by $F(a, b ; c ; z)$. Thus for complex numbers $a, b$ and $c$ with $c \neq 0,-1,-2, \ldots$, and for $|z|<1$,

where, for example,

$$
F(a, b ; c ; z)=\sum_{n=0}^{\infty} \frac{(a)_{n}(b)_{n}}{(c)_{n}} \frac{z^{n}}{n !}
$$

$$
\begin{aligned}
& (a)_{0}=1, \\
& (a)_{n}=a(a+1) \ldots(a+n-1)=\Gamma(a+n) / \Gamma(a) \quad(n \geqq 1) .
\end{aligned}
$$

For brevity we shall write

$$
F^{*}(a, b ; c ; z)=F(a, b ; c ; z) / \Gamma(c) .
$$

Thus, for $|z|<1$ and for any complex numbers $a, b$ and $c$

$$
F^{*}(a, b ; c ; z)=\sum_{n=0}^{\infty} \frac{(a)_{n}(b)_{n}}{\Gamma(c+n)} \frac{z^{n}}{n !}
$$

The restriction $c \neq 0,-1,-2, \ldots$ is no longer necessary since the reciprocal of the gamma function is an entire function. $\mathrm{F}^{*}(a, b ; c ; z)$ as defined by $(2.2)$ is an entire function of $a, b$ and $c$, and an analytic function of $z$ for $|z|<1$.

We shall require values of $F(a, b ; c ; z)$ for $z$ on the negative real axis. We therefore extend $F^{*}(a, b ; c ; z)$ to the half-plane $\operatorname{Re} z<\frac{1}{2}$ using one of Kummer's relations ((2), p. 105)

$$
F^{*}(a, b ; c ; z)=(1-z)^{-a} F^{*}\left(a, c-b ; c ; \frac{z}{z-1}\right)
$$

using the principal branch of $(1-z)^{-a}$. The extended function is an entire function of $a, b$ and $c$, and an analytic function of $z$ for $\operatorname{Re} z<\frac{1}{2}$. Also, by (2.2) and analytic continuation,

for $\operatorname{Re} z<\frac{1}{2}$.

$$
F^{*}(a, b ; c ; z)=F^{*}(b, a ; c ; z)
$$

To discuss the operator $H_{1}(a, b ; c ; m)$ we require the following result.

Lemma 2.1. Let $a, b$ and $c$ be complex numbers and let $\delta>0$. Then there exists a constant $M$, independent of $v$, such that, for $0<v<1$, the four expressions

$$
\begin{aligned}
& \left|F^{*}\left(a, b ; c ; 1-1 / v^{m}\right)\right|,\left|\frac{\partial}{\partial a} F^{*}\left(a, b ; c ; 1-1 / v^{m}\right)\right| \\
& \left|\frac{\partial}{\partial b} F^{*}\left(a, b ; c ; 1-1 / v^{m}\right)\right|,\left|\frac{\partial}{\partial c} F^{*}\left(a, b ; c ; 1-1 / v^{m}\right)\right|
\end{aligned}
$$

are all less than or equal to $M v^{\min (m \operatorname{Re} a, m \operatorname{Re} b)-\delta \text {. }}$ 
Proof. The proof of this result for $m=1$ is given in (8). The general result then follows easily.

\section{3}

We now proceed to the discussion of $H_{1}(a, b ; c ; m)$ on $F_{p, \mu}$. We recall that for $\operatorname{Re} c>0, m>0$ and suitable functions $\phi, H_{1}(a, b ; c ; m) \phi$ is defined, for $0<x<\infty$, by

$H_{1}(a, b ; c ; m) \phi(x)=\int_{0}^{x}\left(x^{m}-t^{m}\right)^{c-1} F^{*}\left(a, b ; c ; 1-x^{m} / t^{m}\right) m t^{m-1} \phi(t) d t$.

To begin with, we note Then

Lemma 3.1. Let $\operatorname{Re} c>0,-\operatorname{Re} \mu-m+1 / p<\min (m \operatorname{Re} a, m \operatorname{Re} b), \phi \in F_{p, \mu}$.

(i) the integral (3.1) for $H_{1}(a, b ; c ; m) \phi(x)$ exists and defines a continuous function of $x$ on $(0, \infty)$,

(ii) for each fixed $x \in(0, \infty), H_{1}(a, b ; c ; m) \phi(x)$ is an analytic function of the (single) variables $a, b, c$ in the regions $-\operatorname{Re} \mu-m+1 / p<\min (m \operatorname{Re} a$, $m \operatorname{Re} b)$ and $\operatorname{Re} c>0$.

Proof. For $x \in(0, \infty)$ we have, from (3.1),

$H_{1}(a, b ; c ; m) \phi(x)=x^{m c} \int_{0}^{1}\left(1-v^{m}\right)^{c-1} F^{*}\left(a, b ; c ; 1-1 / v^{m}\right) m v^{m-1} \phi(x v) d v$.

By Lemma 2.1 above and Lemma 2.2 of (9), for any given $\delta>0$, there exists $M$ independent of $v \in(0,1)$ such that

$$
\begin{aligned}
& \left|\left(1-v^{m}\right)^{c-1} F^{*}\left(a, b ; c ; 1-1 / v^{m}\right) m v^{m-1} \phi(x v)\right| \\
& \leqq M\left(1-v^{m}\right)^{\operatorname{Re} c-1} v^{\min } \quad(m \operatorname{Re} a, m \operatorname{Re} b)-\delta v^{m-1}(x v)^{\operatorname{Re} \mu-1 / p}
\end{aligned}
$$

for $v \in(0,1)$. Under the given conditions on the parameters, the right-hand side of this inequality is an integrable function of $v$ over $(0,1)$ provided $\delta$ is chosen sufficiently close to 0 . Hence the integral on the right of (3.2) converges uniformly on compact subsets of $(0, \infty)$ and (i) follows. (ii) follows similarly using Lemma 2.1 since, under the given conditions, we may differentiate under the integral sign in (3.2) with respect to $a, b$ or $c$.

The main use of Lemma 3.1 is in resolving a minor technical detail below. The information it gives turns out to be relatively little as we shall see later.

To obtain a full description of the mapping properties of $H_{1}(a, b ; c ; m)$ on $F_{p, \mu}$ we proceed to establish a connection with fractional integrals.

\section{Lemma 3.2. Let}

$\operatorname{Re} \alpha>0, \operatorname{Re} \beta>0,-\operatorname{Re} \mu-m+1 / p<\min (m \operatorname{Re} \xi, m \operatorname{Re} \eta), \phi \in F_{p, \mu}$.

Then, for $x>0$,

$$
I_{x^{m}}^{\eta, \alpha} I_{x^{m}}^{\xi, \beta} \phi(x)=x^{-m \eta-m \alpha} H_{1}(\xi+\beta-\eta, \beta ; \alpha+\beta ; m) x^{m \eta-m \beta} \phi(x) .
$$




\section{Proof.}

$$
\begin{aligned}
& I_{x^{m}}^{\eta, \alpha} I_{x^{m}}^{\xi, \beta} \phi(x) \\
& =\frac{m x^{-m \eta-m x}}{\Gamma(\alpha)} \int_{0}^{x}\left(x^{m}-u^{m}\right)^{z-1} u^{m \eta+m-1} d u \frac{m u^{-m \xi-m \beta}}{\Gamma(\beta)} \int_{0}^{u}\left(u^{m}-t^{m}\right)^{\beta-1} t^{m \xi+m-1} \phi(t) d t .
\end{aligned}
$$

By Lemma 2.2 of (9), there is a constant $M:|\phi(t)| \leqq M t^{\operatorname{Re} \mu-1 / p}(0<t<\infty)$. It then follows easily that the repeated integral is absolutely convergent under the given conditions on the parameters. By Fubini's Theorem we may justifiably invert the order of integration to obtain

$$
\frac{m x^{-m \eta-m \alpha}}{\Gamma(\alpha) \Gamma(\beta)} \int_{0}^{x} t^{m \xi+m-1} \phi(t) d t \int_{t}^{x}\left(x^{m}-u^{m}\right)^{\alpha-1}\left(u^{m}-t^{m}\right)^{\beta-1} u^{m \eta-m \xi-m \beta} m u^{m-1} d u .
$$

Under the substitution $w=\left(u^{m}-t^{m}\right) /\left(x^{m}-t^{m}\right)$ the inner integral becomes

$$
\begin{aligned}
& \left(x^{m}-t^{m}\right)^{\alpha+\beta-1} t^{m \eta-m \xi-m \beta} \int_{0}^{1}(1-w)^{\alpha-1} w^{\beta-1}\left[1-w\left(1-x^{m} / t^{m}\right)\right]^{\eta-\xi-\beta} d w \\
& =\frac{\Gamma(\alpha) \Gamma(\beta)}{\Gamma(\alpha+\beta)}\left(x^{m}-t^{m}\right)^{\alpha+\beta-1} t^{m \eta-m \xi-m \beta} F\left(\xi+\beta-\eta, \beta ; \alpha+\beta ; 1-x^{m} / t^{m}\right)
\end{aligned}
$$

using_Euler's Integral, formula (10) on p. 59 of (2). Finally, therefore,

$$
\begin{aligned}
& I_{x^{m}}^{\eta, \alpha} I_{x^{m}}^{\xi, \beta} \phi(x) \\
& =\frac{x^{-m \eta-m x}}{\Gamma(\alpha+\beta)} \int_{0}^{x}\left(x^{m}-t^{m}\right)^{\alpha+\beta-1} F\left(\xi+\beta-\eta, \beta ; \alpha+\beta ; 1-x^{m} / t^{m}\right) m t^{m-1} t^{m \eta-m \beta} \phi(t) d t \\
& =x^{-m \eta-m x} H_{1}(\xi+\beta-\eta, \beta ; \alpha+\beta ; m) x^{m \eta-m \beta} \phi(x) \text { as required. }
\end{aligned}
$$

This completes the proof.

Corollary 3.3. Let

$\operatorname{Re} c>\operatorname{Re} b>0,-\operatorname{Re} \mu-m+1 / p<\min (m \operatorname{Re} a, \mathrm{~m} \operatorname{Re} b), \phi \in F_{p, \mu}$. Then for $x>0$,

$$
H_{1}(a, b ; c ; m) \phi(x)=I_{x^{m}}^{c-b} x^{-m a} I_{x^{m}}^{b} x^{m a} \phi(x) .
$$

Proof. In Lemma 3.2, we take $\alpha=c-b, \beta=b, \xi=\eta+a-b$, and replace $\mu$ and $\phi(x)$ by $\mu-m \eta+m b$ and $x^{-m \eta+m b} \phi(x)$. The conditions in Corollary 3.3 then imply that the conditions of Lemma 3.2 are satisfied so that (after a slight rearrangement)

$$
H_{1}(a, b ; c ; m) \phi(x)=x^{m \eta+m c-m b} I_{x^{m}}^{\eta, c-b} I_{x^{m}}^{\eta+a-b, b} x^{-m \eta+m b} \phi(x)
$$

for $x>0$. The free parameter $\eta$ disappears when we rewrite (3.5) in terms of the inhomogeneous operators $I_{x^{m}}^{\alpha}$ and (3.4) follows almost immediately.

In (9), we extended the operators $I_{x^{m}}^{\eta, \alpha}$ and $I_{x^{m}}^{\alpha}$ on $F_{p, \mu}$ using analytic continuation to values of $\alpha$ with $\operatorname{Re} \alpha \leqq 0$. The right-hand side of (3.4) therefore has a meaning even if the condition $\operatorname{Re} c>\operatorname{Re} b>0$ is removed. We must 
however retain the restriction $-\operatorname{Re} \mu-m+1 / p<\min (m \operatorname{Re} a, m \operatorname{Re} b)$; for details, we refer the reader to Section 3 of (9). We now use (3.4) to extend the definition of $H_{1}(a, b ; c ; m)$ on $F_{p, \mu}$ removing the restriction $\operatorname{Re} c>0$.

Definition 3.4. For $\phi \in F_{p, \mu}$ with $-\operatorname{Re} \mu-m+1 / p<\min (m \operatorname{Re} a, m \operatorname{Re} b)$, we define $H_{1}(a, b ; c ; m) \phi$ by

$$
H_{1}(a, b ; c ; m) \phi(x)=I_{x^{m}}^{c-b} x^{-m a} I_{x^{m}}^{b} x^{m a} \phi(x) \quad(x>0) .
$$

Note. We must be a little careful here and check that this new definition coincides with the original where both make sense, namely where the above conditions are satisfied and $\operatorname{Re} c>0$. Certainly this is the case if $\operatorname{Re} c>\operatorname{Re} b>0$ by Corollary 3.3. However, under the given conditions both sides are analytic functions of $b$ (using Lemma 3.1 for the left-hand side). The principle of analytical continuation then gives the desired result for $\operatorname{Re} c>0$.

With Definition 3.4 available, we can now use results in (9) to obtain the mapping properties of $H_{1}(a, b ; c ; m)$.

Theorem 3.5. If $-\operatorname{Re} \mu-m+1 / p<\min (m \operatorname{Re} a, m \operatorname{Re} b), H_{1}(a, b ; c ; m)$ is a continuous linear mapping of $F_{p, \mu}$ into $F_{p, \mu+m c}$.

If, in addition, $-\operatorname{Re} \mu-m+1 / p<\min (m \operatorname{Re} c, m \operatorname{Re}(a+b)), H_{1}(a, b ; c ; m)$ is an isomorphism of $F_{p, \mu}$ onto $F_{p, \mu+m c}$ and, for $\psi \in F_{p, \mu+m c}$,

$$
\left[H_{1}(a, b ; c ; m)\right]^{-1} \psi(x)=x^{-m a} I_{x^{m}}^{-b} x^{m a} I_{x^{m}}^{b-c} \psi(x) .
$$

Notice that, in the above theorem, " isomorphism " is used in the sense of Zemanian (10, p. 27).

Proof. Let $\phi \in F_{p, \mu}$. Then $x^{m a} \phi \in F_{p, \mu+m a}$ (Theorem 2.6 (i) of (9)). Since $\operatorname{Re}(\mu+m a)+m>1 / p$, we can apply Theorem 3.6 of (9) with $\mu$ and $\phi$ replaced by $\mu+m a$ and $x^{m a} \phi$ respectively to deduce that $I_{x^{m}}^{b} x^{m a} \phi \in F_{p, \mu+m a+m b}$. Then

$$
x^{-m a} I_{x^{m}}^{b} x^{m a} \phi \in F_{p, \mu+m b}
$$

(Theorem 2.6 (i) of (9)). Finally, since $\operatorname{Re}(\mu+m b)+m>1 / p$, we can again apply Theorem 3.6 of (9) with $\mu$ and $\phi$ replaced by $\mu+m b$ and $x^{-m a} I_{x^{m}}^{b} x^{m a} \phi$ respectively to obtain $I_{x^{m}}^{c-b} x^{-m a} I_{x^{m}}^{b} x^{m a} \phi \in F_{p, \mu+m c}$. Further, the theorems quoted above also show that $H_{1}(a, b ; c ; m)$, being the composition of four continuous linear mappings, is itself a continuous linear mapping of $F_{p, \mu}$ into $F_{p, \mu+m c}$. The second part of the theorem is proved similarly; the extra conditions are needed to ensure the invertibility of $I_{x^{m}}^{b}$ and $I_{x^{m}}^{c-b}$ (see Theorem 3.6 of (9) again). We note that it is also possible to prove the theorem by using (3.5) above instead of Theorem 3.5 of (9).

One interesting consequence is

Corollary 3.6. If

$\psi \in F_{p, \mu+m c}$ and $-\operatorname{Re} \mu-m+1 / p<\min (m \operatorname{Re} a, m \operatorname{Re} b, m \operatorname{Re} c, m \operatorname{Re}(a+b))$, then for $x>0$,

$$
\left[H_{1}(a, b ; c ; m)\right]^{-1} \psi(x)=x^{-m a} H_{1}(-a, b-c ;-c ; m) x^{m a} \psi(x) .
$$


Proof. This follows immediately from (3.6) and Definition 3.4; in the latter $a, b, c, \mu$ and $\phi(x)$ have to be replaced by $-a, b-c,-c, \mu+m a+m c$ and $x^{m a} \phi(x)$ respectively. then

Next we remark that if $\phi \in F_{p, \mu}$ and $-\operatorname{Re} \mu-m+1 \mid p<\min (m \operatorname{Re} a, m \operatorname{Re} b)$,

$$
H_{1}(a, b ; c ; m) \phi=H_{1}(b, a ; c ; m) \phi .
$$

Indeed, this follows for $\operatorname{Re} c>0$ from (2.4) and (3.1) and then in general by analytic continuation. It is hardly surprising that the restrictions on the parameters are symmetric in $a$ and $b$. However, the right-hand side in Definition 3.4 is not symmetric in $a$ and $b$. We exploit this lack of symmetry to establish the second index law for the operators $I_{x^{m}}^{\alpha}$ on the spaces $F_{p, \mu}$. There are various ways of stating this of which we choose the following (see (3.13) of (9)).

Theorem 3.7. If $\alpha+\beta+\gamma=0$ and if $\phi \in F_{p, \mu}$ where

then, for $x>0$,

$$
-\operatorname{Re} \mu-m+1 / p<\min (0, m \operatorname{Re} \gamma)
$$

$$
x^{m \alpha} I_{x^{m}}^{\beta} x^{m \gamma} \phi(x)=I_{x^{m}}^{-y} x^{-m \beta} I_{x^{m}}^{-\alpha} \phi(x) .
$$

Proof. Under the given conditions we apply (3.8) with $a, b, c, \mu$ and $\phi(x)$ replaced by $-\alpha, \beta, \beta, \mu-m \beta$ and $x^{-m \beta} \phi(x)$ respectively. To do this we need

or

$$
-\operatorname{Re}(\mu-m \beta)-m+1 / p<\min (-m \operatorname{Re} \alpha, m \operatorname{Re} \beta)
$$

$$
-\operatorname{Re} \mu-m+1 / p<\min (-m \operatorname{Re}(\alpha+\beta), 0)=\min (m \operatorname{Re} \gamma, 0)
$$

and this is the case by hypothesis. Hence (3.8) gives

$$
H_{1}(-\alpha, \beta ; \beta ; m)\left(x^{-m \beta} \phi\right)=H_{1}(\beta,-\alpha ; \beta ; m)\left(x^{-m \beta} \phi\right) .
$$

Using Definition 3.4 then gives

$$
I_{x^{m}}^{0} x^{m \alpha} I_{x^{m}}^{\beta} x^{-m \alpha} x^{-m \beta} \phi=I_{x^{m}}^{\beta+\alpha} x^{-m \beta} I_{x^{m}}^{-\alpha} x^{m \beta} x^{-m \beta} \phi .
$$

Theorem 3.6 shows that under the given circumstances, $\phi \in F_{p, \mu}$ implies that $x^{m \alpha} I_{x}^{\beta} x^{-m \alpha-m \beta} \phi \in F_{p, \mu}$ and, in addition, $I_{x^{m}}^{0}$ is the identity operator on $F_{p, \mu}$. Hence, putting $\gamma=-\alpha-\beta$ in (3.10) gives (3.9) as required.

4

We now introduce three more integral operators related to $H_{1}(a, b ; c ; m)$. For any complex numbers $a$ and $b, \operatorname{Re} c>0(m>0$ as usual) and suitable functions $\phi$, we define $H_{2}(a, b ; c ; m) \phi$ by

$H_{2}(a, b ; c ; m) \phi(x)=\int_{0}^{x}\left(x^{m}-t^{m}\right)^{c-1} F^{*}\left(a, b ; c ; 1-t^{m} / x^{m}\right) m t^{m-1} \phi(t) d t$,

where $x>0$. Proceeding as in $(5$, p. 195), we deduce that, for $x>0$,

$$
H_{2}(a, b ; c ; m) \phi(x)=x^{m a} H_{1}(a, c-b ; c ; m) x^{-m a} \phi(x)
$$


whenever either side exists. In particular, if we apply Theorem 3.5 with $b, \mu$ and $\phi(x)$ replaced by $c-b, \mu-m a$ and $x^{-m a} \phi(x)$ respectively we find that

$$
\phi \in F_{p, \mu} \Rightarrow H_{2}(a, b ; c ; m) \phi \in F_{p, \mu+m c}
$$

provided that $-\operatorname{Re}(\mu-m a)-m+1 / p<\min (m \operatorname{Re} a, m \operatorname{Re}(c-b))$ and $\operatorname{Re} c>0$. However, the right-hand side of (4.2) is meaningful even without the restriction $\operatorname{Re} c>0$. We can therefore use (4.2) to extend the definition of $H_{2}(a, b ; c ; m)$ on $F_{p, \mu}$.

Definition 4.1. For $\phi \in F_{p, \mu}$ with $-\operatorname{Re} \mu-m+1 / p<\min (0, m \operatorname{Re}(c-a-b))$, we define $H_{2}(a, b ; c ; m) \phi$ by

$$
H_{2}(a, b ; c ; m) \phi(x)=x^{m a} H_{1}(a, c-b ; c ; m) x^{-m a} \phi(x) \quad(x>0) .
$$

In view of the preceding remarks, the definition is meaningful and agrees with (4.1) when, in addition, $\operatorname{Re} c>0$.

Using Theorem 3.5, we can easily prove

Theorem 4.2. If $-\operatorname{Re} \mu-m+1 / p<\min (0, m \operatorname{Re}(c-a-b))$, then

$$
\mathrm{H}_{2}(a, b ; c ; m)
$$

is a continuous linear mapping of $F_{p, \mu}$ into $F_{p, \mu+m c}$ and, for $\phi \in F_{p, \mu}$,

$$
H_{2}(a, b ; c ; m) \phi(x)=x^{m a} I_{x^{m}}^{b} x^{-m a} I_{x^{m}}^{c-b} \phi(x) \quad(x>0) .
$$

If, in addition,

$$
-\operatorname{Re} \mu-m+1 / p<\min (m \operatorname{Re}(c-a), m \operatorname{Re}(c-b)) \text {, }
$$

then $\mathrm{H}_{2}(a, b ; c ; m)$ is an isomorphism of $F_{p, \mu}$ onto $F_{p, \mu+m c}{ }^{\text {and }}$ and for any $\psi \in F_{p, \mu+m c}$, the equation

$$
H_{2}(a, b ; c ; m) \phi=\psi
$$

has a unique solution $\phi \in F_{p, \mu}$ given by

This leads to

$$
\phi(x)=I_{x^{m}}^{b-c} x^{m a} I_{x^{m}}^{-b} x^{-m a} \psi(x) \quad(x>0) .
$$

\section{Corollary 4.3. Let}

$-\operatorname{Re} \mu-m+1 / p<\min (0, m \operatorname{Re}(c-a-b), m \operatorname{Re}(c-a), m \operatorname{Re}(c-b)), \phi \in F_{p, \mu}$ and $\psi \in F_{p, \mu+m c}$. Then

$$
H_{2}(a, b ; c ; m) \phi=\psi \Leftrightarrow \phi=H_{1}(-a,-b ;-c ; m) \dot{\psi} .
$$

Proof. Since by hypothesis,

$$
-\operatorname{Re}(\mu+m c)-m+1 / p
$$

$<\min (m \operatorname{Re}(-c), m \operatorname{Re}(-a-b), m \operatorname{Re}(-a), m \operatorname{Re}(-b))$, we can apply Definition 3.4 with $a, b, c, \mu$ and $\phi(x)$ replaced by $-a,-b,-c$, $\mu+m c$ and $\psi(x)$ respectively to obtain

$$
H_{1}(-a,-b ;-c ; m) \psi(x)=I_{x^{m}}^{b-c} x^{m a} I_{x^{m}}^{-b} x^{-m a} \psi(x) \quad(x>0) .
$$

The result follows at once from Theorem 4.2 and in particular (4.5). 
Our other two operators are the adjoints of $H_{1}(a, b ; c ; m)$ and $H_{2}(a, b ; c ; m)$ and reduce in the case $m=1$ to those studied by Love in (6). For complex numbers $a$ and $b, \operatorname{Re} c>0$ and suitable functions $\phi$, we define $H_{3}(a, b ; c ; m) \phi$ and $H_{4}(a, b ; c ; m) \phi$ on $(0, \infty)$ by

$$
\begin{aligned}
& H_{3}(a, b ; c ; m) \phi(x)=m x^{m-1} \int_{x}^{\infty}\left(t^{m}-x^{m}\right)^{c-1} F^{*}\left(a, b ; c ; 1-x^{m} / t^{m}\right) \phi(t) d t \\
& H_{4}(a, b ; c ; m) \phi(x)=m x^{m-1} \int_{x}^{\infty}\left(t^{m}-x^{m}\right)^{c-1} F^{*}\left(a, b ; c ; 1-t^{m} / x^{m}\right) \phi(t) d t .
\end{aligned}
$$

As we might expect, it is possible to express these operators in terms of operators of the form $K_{x^{m}}^{\eta, \alpha}$ or $K_{x^{m}}^{\alpha}$. Indeed, proceeding as in Lemma 3.2, we find that for $x>0$,

$$
K_{x m}^{\xi ; \beta} K_{x m}^{\eta, \alpha} \phi(x)=x^{m \xi-m+1} H_{3}(\xi+\beta-\eta, \alpha ; \alpha+\beta ; m) x^{-m \xi-m \beta-m a+m-1} \phi(x)
$$

provided $\operatorname{Re} \alpha>0, \operatorname{Re} \beta>0, \phi \in F_{p, \mu}$ and $\operatorname{Re} \mu-1 / p<\min (m \operatorname{Re} \xi, m \operatorname{Re} \eta)$. It follows, as before, that

$$
\begin{aligned}
& H_{3}(a, b ; c ; m) \phi(x) \\
& \quad=x^{-m \eta-m a-m b+m c+m-1} K_{x^{m}}^{a+b-c+\eta, c-b} K_{x^{m}}^{\eta, b} x^{m \eta+m a+m b-m+1} \phi(x) !
\end{aligned}
$$

or

provided

$$
H_{3}(a, b ; c ; m) \phi(x)=x^{m-1} K_{x^{m}}^{c-b} x^{-m a} K_{x^{m}}^{b} x^{m a-m+1} \phi(x)
$$

$\operatorname{Re} c>\operatorname{Re} b>0, \phi \in F_{p, \mu}$ and $\operatorname{Re} \mu-m+1 / q<\min (-m \operatorname{Re} c,-m \operatorname{Re}(a+b))$. As before, we can use (4.9) to extend the definition of $H_{3}(a, b ; c ; m)$ on $F_{p, \mu}$ removing the restriction $\operatorname{Re} c>\operatorname{Re} b>0$.

Definition 4.4. For $\phi \in F_{p, \mu}$ and

$$
\operatorname{Re} \mu-m+1 / q<\min (-m \operatorname{Re} c,-m \operatorname{Re}(a+b)) \text {, }
$$

define $H_{3}(a, b ; c ; m) \phi$ by

$$
H_{3}(a, b ; c ; m) \phi(x)=x^{m-1} K_{x^{m}}^{c-b} x^{-m a} K_{x^{m}}^{b} x^{m a-m+1} \phi(x) \quad(x>0) .
$$

By analytic continuation, this definition coincides with (4.6) when in addition $\operatorname{Re} c>0$. Using the mapping properties of $K_{x^{m}}^{\eta, \alpha}$ or $K_{x^{m}}^{\alpha}$ derived in Theorems 3.7 and 3.8 of (9), we immediately obtain

Theorem 4.5. If

$$
\operatorname{Re} \mu-m+1 / q<\min (-m \operatorname{Re} c,-m \operatorname{Re}(a+b)), H_{3}(a, b ; c ; m)
$$

is a continuous linear mapping of $F_{p, \mu}$ into $F_{p, \mu+m c}$. If, in addition,

$$
\operatorname{Re} \mu-m+1 / q<\min (-m \operatorname{Re} a,-m \operatorname{Re} b), H_{3}(a, b ; c ; m)
$$

is an isomorphism of $F_{p, \mu}$ onto $F_{p, \mu+m c}$ and, for any $\psi \in F_{p, \mu+m c}$, the equation

$$
H_{3}(a, b ; c ; m) \phi=\psi
$$


has a unique solution $\phi \in F_{p, \mu}$ given by

$$
\phi(x)=x^{-m a+m-1} K_{x^{m}}^{-b} x^{m a} K_{x^{m}}^{b-c} x^{-m+1} \psi(x) \quad(x>0) .
$$

As regards $H_{4}(a, b ; c ; m)$, we may use (4.6) and (4.7) and proceed as in ((6), pp. 1073-4) to show that for $\operatorname{Re} c>0$,

$$
H_{4}(a, b ; c ; m) \phi(x)=x^{m a} H_{3}(a, c-b ; c ; m) x^{-m a} \phi(x)
$$

whenever either side exists. In particular, from Theorem 4.5, (4.12) is valid if $\phi \in F_{p, \mu}, \operatorname{Re} \mu-m+1 / q<\min (m \operatorname{Re}(a-c), m \operatorname{Re}(b-c))$ and $\operatorname{Re} c>0$. The right-hand side is meaningful even without the restriction $\operatorname{Re} c>0$ and we can use (4.12) to extend the definition of $H_{4}(a, b ; c ; m)$ on $F_{p, \mu}$.

Definition 4.6. For $\phi \in F_{p, \mu}$ and

$$
\operatorname{Re} \mu-m+1 / q<\min (m \operatorname{Re}(a-c), m \operatorname{Re}(b-c)),
$$

define $H_{4}(a, b ; c ; m) \phi$ on $(0, \infty)$ by

$$
H_{4}(a, b ; c ; m) \phi(x)=x^{m a} H_{3}(a, c-b ; c ; m) x^{-m a} \phi(x) .
$$

The definition agrees with (4.7) when also $\operatorname{Re} c>0$.

Using Definition 4.4 and Theorem 4.5 we obtain

Theorem 4.7. If

$\operatorname{Re} \mu-m+1 / q<\min (m \operatorname{Re}(a-c), m \operatorname{Re}(b-c)), H_{4}(a, b ; c ; m)$ is a continuous linear mapping of $F_{p, \mu}$ into $F_{p, \mu+m c}$ and, for $\phi \in F_{p, \mu}$,

$$
H_{4}(a, b ; c ; m) \phi(x)=x^{m a+m-1} K_{x^{m}}^{b} x^{-m a} K_{x^{m}}^{c-b} x^{-m+1} \phi(x) \quad(x>0) .
$$

If, in addition, $\operatorname{Re} \mu-m+1 / q<\min (0, m \operatorname{Re}(a+b-c)), H_{4}(a, b ; c ; m)$ is an isomorphism of $F_{p, \mu}$ onto $F_{p, \mu+m c}$ and, for any $\psi \in F_{p, \mu+m c}$, the equation

$$
H_{4}(a, b ; c ; m) \phi=\psi
$$

has a unique solution $\phi \in F_{p, \mu}$ given by

$$
\phi(x)=x^{m-1} K_{x^{m}}^{b-c} x^{m a} K_{x^{m}}^{-b} x^{-m a-m+1} \psi(x) \quad(x>0) .
$$

Comparing (4.15) with Definition 4.4 produces the following analogue of Corollary 4.3 .

Corollary 4.8. Let

$\operatorname{Re} \mu-m+1 / q<\min (0, m \operatorname{Re}(a+b-c), m \operatorname{Re}(a-c), m \operatorname{Re}(b-c)), \phi \in F_{p, \mu}$ and $\psi \in F_{p, \mu+m c}$. Then

$$
H_{4}(a, b ; c ; m) \phi=\psi \Leftrightarrow \phi=H_{3}(-a,-b ;-c ; m) \psi .
$$

To conclude this section, we state the second index law for the operators $K_{x^{m}}^{\alpha}$ analogous to Theorem 3.7.

Theorem 4.9. If $\alpha+\beta+\gamma=0$ and if $\phi \in F_{p, \mu}$ where

$$
\operatorname{Re} \mu-1 / p<\min (0, m \operatorname{Re} \gamma),
$$


then, for $x>0$,

$$
x^{m y} K_{x^{m}}^{\beta} x^{m x} \phi(x)=K_{x^{m}}^{-\alpha} x^{-m \beta} K_{x^{m}}^{-y} \phi(x) .
$$

This can be proved by observing that under appropriate conditions

$$
H_{4}(a, b ; c ; m) \phi=H_{4}(b, a ; c ; m) \phi
$$

and proceeding as in Theorem 3.7 making use of Theorem 3.8 of (9).

5

We are now going to discuss the operators $H_{i}(a, b ; c ; m)(i=1,2,3,4)$ relative to the spaces $F_{p, \mu}^{\prime}$.

We consider first $H_{1}(a, b ; c ; m)$ and to motivate our definition we deal with regular functionals. We require the following by

Definition 5.1. For each complex number $\mu$ and $1 \leqq p \leqq \infty$, we define $L_{\mu}^{p}$

$$
L_{\mu}^{p}=\left\{f: x^{-\mu} f(x) \in L^{p}(0, \infty)\right\} .
$$

We can turn $L_{\mu}^{p}$ into a Banach space by introducing the norm \|\|$_{p, \mu}$ defined by

$$
\|f\|_{p, \mu}=\left\|x^{-\mu} f(x)\right\|_{p} \quad\left(f \in L_{\mu}^{p}\right)
$$

where \|\|$_{p}$ denotes the usual norm on $L^{p}(0, \infty)$.

If $f \in L_{\mu}^{p}$ and $\operatorname{Re} c>0$ we can define $H_{1}(a, b ; c ; m) f$ using (1.5), with $\phi$ replaced by $f$, under appropriate conditions on $a, b$ and $\mu$. Indeed we can prove

\section{Lemma 5.2. If}

$\operatorname{Re} c>0$ and $-\operatorname{Re} \mu-m+1 / p<\min (m \operatorname{Re} a, m \operatorname{Re} b), H_{1}(a, b ; c ; m)$ (as given by (1.5)) is a continuous linear mapping of $L_{\mu}^{p}$ into $L_{\mu+m c}^{p}$.

Proof. Using (1.5) and putting $t=x v$ we have (see (3.2)) for $x>0$,

$$
H_{1}(a, b ; c ; m) f(x)=x^{m c} \int_{0}^{1}\left(1-v^{m}\right)^{c-1} F^{*}\left(a, b ; c ; 1-1 / v^{m}\right) m v^{m-1} f(x v) d v .
$$

For simplicity write $d=\min (m \operatorname{Re} a, m \operatorname{Re} b)$ and choose $\delta>0$ such that $-\operatorname{Re} \mu-m+1 / p<d-\delta$. Applying Lemma 2.1, there exists a constant $M$ such that

$\left|x^{-\mu-m c} H_{1}(a, b ; c ; m) f(x)\right|$

$$
\begin{aligned}
& \leqq M \int_{0}^{1}\left(1-v^{m}\right)^{\operatorname{Re} c-1} v^{d-\delta+\operatorname{Re} \mu} m v^{m-1}\left|(x v)^{-\mu} f(x v)\right| d v \\
& =M \Gamma(c) I_{x^{\prime m}}^{1 / m(d-\delta+\operatorname{Re} \mu), \operatorname{Re} c}\left(\left|x^{-\mu} f(x)\right|\right)
\end{aligned}
$$

(see (3.3) of $(9))=g(x)$ say. Then

$$
\left|x^{-\mu-m c} H_{1}(a, b ; c ; m) f(x)\right| \leqq g(x) .
$$


Now since $(d-\delta+\operatorname{Re} \mu)+m>1 / p$, we can apply an extension of a result of Kober (4, Theorem 2 (i)) to deduce that, since $\left|x^{-\mu} f(x)\right| \in L^{p}(0, \infty), g \in L^{p}(0, \infty)$ and there is a constant $K$ independent of $f$ such that

$$
\|g(x)\|_{p} \leqq K\left\|x^{-\mu} f(x)\right\|_{p} .
$$

Kober's result deals with the case $m=1, \mu=0$. The general case follows easily by a simple change of variable; compare Lemma 3.1 of (9). Now (5.1), (5.2) give

$\left\|x^{-\mu-m c} H_{1}(a, b ; c ; m) f(x)\right\|_{p} \leqq K\left\|x^{-\mu} f(x)\right\|_{p}$

$$
\Rightarrow\left\|H_{1}(a, b ; c ; m) f(x)\right\|_{p, \mu+m c} \leqq K\|f(x)\|_{p, \mu}
$$

and the result follows.

Now let $f \in L_{-\mu}^{q}$. Then $f$ generates a regular functional, $\tau f$ say, in the space $F_{p, \mu}^{\prime}$ according to the formula

$$
(\tau f, \phi)=\int_{0}^{\infty} f(x) \phi(x) d x \quad\left(\phi \in F_{p, \mu}\right)
$$

the integral on the right being absolutely convergent by Hölder's inequality. If $\operatorname{Re} c>0$ and $\operatorname{Re} \mu-m+1 / q<\min (m \operatorname{Re} a, m \operatorname{Re} b)$, Lemma 5.2 shows that $H_{1}(a, b ; c ; m) f$ exists and belongs to $L_{-\mu+m c}^{q}$. Hence $H_{1}(a, b ; c ; m) f$ generates a regular functional $\tau H_{1}(a, b ; c ; m) f \in F_{p, \mu-m c}^{\prime}$ according to the formula

$\left(\tau H_{1}(a, b ; c ; m) f, \phi\right)=\int_{0}^{\infty} H_{1}(a, b ; c ; m) f(x) \phi(x) d x \quad\left(\phi \in F_{p, \mu-m c}\right)$.

It seems reasonable that we should define $H_{1}(a, b ; c ; m)$ on the space $F_{p, \mu}^{\prime}$ in such a way that if the above conditions are satisfied,

$$
H_{1}(a, b ; c ; m) \tau f=\tau H_{1}(a, b ; c ; m) f .
$$

Note. No confusion should arise from using the same symbol $H_{1}(a, b ; c ; m)$ for the operator on $F_{p, \mu}^{\prime}$ as well as the operator on $F_{p, \mu}$.

(5.5) implies that if $\operatorname{Re} c>0, \operatorname{Re} \mu-m+1 / q<\min (m \operatorname{Re} a, m \operatorname{Re} b)$, $H_{1}(a, b ; c ; m)$ maps regular functionals in $F_{p, \mu}^{\prime}$ into regular functionals in $F_{p, \mu-m c}^{\prime}$. Further if $\phi \in F_{p, \mu-m c}$, we have

$$
\begin{aligned}
\left(H_{1}(a, b ; c ; m) \tau f, \phi\right)=\left(\tau H_{1}(a, b ; c ; m) f, \phi\right) & \text { by }(5.5) \\
\quad=\int_{0}^{\infty} H_{1}(a, b ; c ; m) f(x) \phi(x) d x & \text { by (5.4) } \\
\quad=\int_{0}^{\infty}\left(\int_{0}^{x}\left(x^{m}-t^{m}\right)^{c-1} F^{*}\left(a, b ; c ; 1-x^{m} / t^{m}\right) m t^{m-1} f(t) d t\right) \phi(x) d x & \text { by }(1.5)
\end{aligned}
$$




$$
\begin{aligned}
& =\int_{0}^{\infty} f(t)\left(m t^{m-1} \int_{t}^{\infty}\left(x^{m}-t^{m}\right)^{c-1} F^{*}\left(a, b ; c ; 1-x^{m} / t^{m}\right) \phi(x) d x\right) d t \\
& =\int_{0}^{\infty} f(t) H_{4}(a, b ; c ; m) \phi(t) d t
\end{aligned}
$$

or, using (5.3),

$$
\left(H_{1}(a, b ; c ; m) \tau f, \phi\right)=\left(\tau f, H_{4}(a, b ; c ; m) \phi\right) .
$$

The inversion of the order of integration above is justified since the integrals involved are absolutely convergent by Lemma 5.2, Theorem 4.7 and Hölder's inequality. The derivation of (5.6) required $\operatorname{Re} c>0$ in order that we could use (1.5) to obtain $H_{1}(a, b ; c ; m) f$. If $\operatorname{Re} c \leqq 0$, the integral in (1.5) will not exist for an arbitrary element $f \in L_{-\mu}^{q}$, nor can we use a formula such as Definition 3.4 unless $f$ has some additional differentiability. Nevertheless, by Theorem 4.7, the right-hand side of (5.6) is still meaningful provided only $\phi \in F_{p, \mu-m c}$ and $\operatorname{Re} \mu-m+1 / q<\min (m \operatorname{Re} a, m \operatorname{Re} b)$. We can even go further and replace $\tau f$ by any functional in $F_{p, \mu}^{\prime}$, regular or not. Hence we are led to the following definition.

Definition 5.3. For $f \in F_{p, \mu}^{\prime}$ and $\operatorname{Re} \mu-m+1 / q<\min (m \operatorname{Re} a, m \operatorname{Re} b)$, we define $H_{1}(a, b ; c ; m) f$ as the member of $F_{p, \mu-m c}^{\prime}$ such that

$$
\left(H_{1}(a, b ; c ; m) f, \phi\right)=\left(f, H_{4}(a, b ; c ; m) \phi\right) \quad\left(\phi \in F_{p, \mu-m c}\right) .
$$

Remark 5.4. No confusion should arise from the use of " $f$ " to denote an arbitrary generalised function as well as a classical function generating the regular functional $\tau f$. Indeed it is often convenient to identify a classical function with the functional it generates, although we shall not do so in this paper.

Since Theorem 4.7 details the mapping properties of $H_{4}(a, b ; c ; m)$ on the spaces $F_{p, \mu}$, we can use standard theorems on adjoints (e.g. Theorems 1.10-1 and $1.10-2$ in (10)) to obtain properties of $H_{1}(a, b ; c ; m)$ on the spaces $F_{p, \mu}^{\prime}$.

Theorem 5.5. If $\operatorname{Re} \mu-m+1 / q<\min (m \operatorname{Re} a, m \operatorname{Re} b), H_{1}(a, b ; c ; m)$ is $a$ continuous linear mapping of $F_{p, \mu}^{\prime}$ into $F_{p, \mu-m c}^{\prime}$ and, for $f \in F_{p, \mu}^{\prime}$,

$$
H_{1}(a, b ; c ; m) f=I_{x^{m}}^{c-b} x^{-m a} I_{x^{m}}^{b} x^{m a} f .
$$

If, in addition, $\operatorname{Re} \mu-m+1 / q<\min (m \operatorname{Re} c, m \operatorname{Re}(a+b)), H_{1}(a, b ; c ; m)$ is an isomorphism of $F_{p, \mu}^{\prime}$ onto $F_{p, \mu-m c}^{\prime}$ and, for any $g \in F_{p, \mu-m c}^{\prime}$, the equation

$$
H_{1}(a, b ; c ; m) f=g
$$

has a unique solution $f \in F_{p, \mu}^{\prime}$ given by

$$
f=x^{-m a} I_{x^{m}}^{-b} x^{m a} I_{x^{m}}^{b-c} g .
$$

Proof. By Theorem 4.7, $H_{4}(a, b ; c ; m)$ is a continuous linear mapping of $F_{p, \mu-m c}$ into $F_{p, \mu}$ under the given conditions; the first statement follows from Theorem 1.10-1 of (10). To establish (5.7), let $f \in F_{p, \mu}^{\prime}, \phi \in F_{p, \mu-m c}$. 
Then

$$
\begin{array}{ll}
\left(H_{1}(a, b ; c ; m) f, \phi\right)=\left(f, H_{4}(a, b ; c ; m) \phi\right) & \text { by Definition } 5.3 \\
=\left(f, x^{m a+m-1} K_{x^{m}}^{b} x^{-m a} K_{x^{m}}^{c-b} x^{-m+1} \phi\right) & \text { by (4.13) } \\
=\left(x^{m a} f, x^{m-1} K_{x^{m}}^{b} x^{-m+1} x^{-m a} x^{m-1} K_{x^{m}}^{c-b} x^{-m+1} \phi\right) & \text { by (2.13) of (9) } \\
=\left(I_{x^{m}}^{b} x^{m a} f, x^{-m a} x^{m-1} K_{x^{m}}^{c-b} x^{-m+1} \phi\right) & \text { by (3.29) of (9) } \\
=\left(x^{-m a} I_{x^{m}}^{b} x^{m a} f, x^{m-1} K_{x^{m}}^{c-b} x^{-m+1} \phi\right) & \text { by (2.13) of (9) } \\
=\left(I_{x^{m}}^{c-b} x^{-m a} I_{x^{m}}^{b} x^{m a} f, \phi\right) & \text { by (3.29) of (9) }
\end{array}
$$

from which (5.7) follows. The two applications of (3.29) of (9) above are valid under the given conditions. The remainder of Theorem 5.5 can be proved similarly.

If we compare Theorems 3.5 and 5.5, we see that the restrictions on the parameters in one are obtained from those in the other by interchanging $p$ and $q$, $\mu$ and $-\mu$. This continues the trend we first mentioned after Theorem 3.9 of (9).

We can handle the other operators similarly. We mention the salient facts and omit proofs.

Consideration of regular functionals leads as before to

Definition 5.6. For $f \in F_{p, \mu}^{\prime}$, we define $H_{i}(a, b ; c ; m) f(i=2,3,4)$ to be the elements of $F_{p, \mu-m c}^{\prime}$ such that, for all $\phi \in F_{p, \mu-m c}$,

$$
\begin{aligned}
& \left(H_{2}(a, b ; c ; m) f, \phi\right)=\left(f, H_{3}(a, b ; c ; m) \phi\right) \\
& \left(H_{3}(a, b ; c ; m) f, \phi\right)=\left(f, H_{2}(a, b ; c ; m) \phi\right) \\
& \left(H_{4}(a, b ; c ; m) f, \phi\right)=\left(f, H_{1}(a, b ; c ; m) \phi\right)
\end{aligned}
$$

(whenever the right-hand sides are meaningful).

Theorem 5.7. If $\operatorname{Re} \mu-m+1 / q<\min (0, m \operatorname{Re}(c-a-b)), H_{2}(a, b ; c ; m)$ is a continuous linear mapping of $F_{p, \mu}^{\prime}$ into $F_{p, \mu-m c}^{\prime}$ and, for $f \in F_{p, \mu}^{\prime}$,

$$
H_{2}(a, b ; c ; m) f=x^{m a} I_{x^{m}}^{b} x^{-m a} I_{x^{m}}^{c-b} f \text {. }
$$

If, in addition, $\operatorname{Re} \mu-m+1 / q<\min (m \operatorname{Re}(c-a), m \operatorname{Re}(c-b)), H_{2}(a, b ; c ; m)$ is an isomorphism of $F_{p, \mu}^{\prime}$ onto $F_{p, \mu-m c}^{\prime}$ and, for each $g \in F_{p, \mu-m c}^{\prime}$, the equation

$$
H_{2}(a, b ; c ; m) f=g
$$

has a unique solution $f \in F_{p, \mu}^{\prime}$ given by

$$
f=H_{1}(-a,-b ;-c ; m) g=I_{x^{m}}^{b-c} x^{m a} I_{x^{m}}^{-b} x^{-m a} g .
$$

Theorem 5.8. If

$$
-\operatorname{Re} \mu-m+1 / p<\min (-m \operatorname{Re} c,-m \operatorname{Re}(a+b)), H_{3}(a, b ; c ; m)
$$

is a continuous linear mapping of $F_{p, \mu}^{\prime}$ into $F_{p, \mu-m c}^{\prime}$ and, for $f \in F_{p, \mu}^{\prime}$,

$$
H_{3}(a, b ; c ; m) f=x^{m-1} K_{x^{m}}^{c-b} x^{-m a} K_{x^{m}}^{b} x^{m a-m+1} f .
$$


If, in addition, $-\operatorname{Re} \mu-m+1 / p<\min (-m \operatorname{Re} a,-m \operatorname{Re} b), H_{3}(a, b ; c ; m)$ is an isomorphism of $F_{p, \mu}^{\prime}$ onto $F_{p, \mu-m c}^{\prime}$ and, for each $g \in F_{p, \mu-m c}^{\prime}$, the equation

$$
H_{3}(a, b ; c ; m) f=g
$$

has a unique solution $f \in F_{p, \mu}^{\prime}$ given by

Theorem 5.9. If

$$
f=x^{-m a+m-1} K_{x^{m}}^{-b} x^{m a} K_{x^{m}}^{b-c} x^{-m+1} g .
$$

$-\operatorname{Re} \mu-m+1 / p<\min (m \operatorname{Re}(a-c), m \operatorname{Re}(b-c)), H_{4}(a, b ; c ; m)$ is a continuous linear mapping of $F_{p, \mu}^{\prime}$ into $F_{p, \mu-m c}^{\prime}$ and, for $f \in F_{p, \mu}^{\prime}$,

$$
H_{4}(a, b ; c ; m) f=x^{m a+m-1} K_{x^{m}}^{b} x^{-m a} K_{x^{m}}^{c-b} x^{-m+1} f .
$$

If, in addition, $-\operatorname{Re} \mu-m+1 / p<\min (0, m \operatorname{Re}(a+b-c)), H_{4}(a, b ; c ; m)$ is an isomorphism of $F_{p, \mu}^{\prime}$ onto $F_{p, \mu-m c}^{\prime}$ and, for each $g \in F_{p, \mu-m c}^{\prime}$, the equation

$$
H_{4}(a, b ; c ; m) f=g
$$

has a unique solution $f \in F_{p, \mu}^{\prime}$ given by

$$
f=H_{3}(-a,-b ;-c ; m) g=x^{m-1} K_{x^{m}}^{b-c} x^{m a} K_{x^{m}}^{-b} x^{-m a-m+1} g .
$$

Finally, we state the second index laws for $I_{x^{m}}^{\alpha}$ and $K_{x^{m}}^{\alpha}$ on $F_{p, \mu}^{\prime}$.

Theorem 5.10. Let $f \in F_{p, \mu}^{\prime}$ and $\alpha+\beta+\gamma=0$.

(i) If $\operatorname{Re} \mu-m+1 / q<\min (0, m \operatorname{Re} \gamma)$, then

$$
x^{m \alpha} I_{x^{m}}^{\beta} x^{m y} f=I_{x^{m}}^{-\gamma} x^{-m \beta} I_{x^{m}}^{-\alpha} f .
$$

(ii) If $-\operatorname{Re} \mu-1 / q<\min (0, m \operatorname{Re} \gamma)$, then

$$
x^{m \gamma} K_{x^{m}}^{\beta} x^{m a} f=K_{x^{m}}^{-\alpha} x^{-m \beta} K_{x^{m}}^{-\gamma} f .
$$

The basis of Theorem 5.10 is the symmetry of the $H_{i}(a, b ; c ; m) f$ between $a$ and $b$ which is inherited from the symmetry of $H_{i}(a, b ; c ; m) \phi$ as exemplified by (3.8) and (4.17).

\section{6}

In this section we compare and contrast our results with those of Love in (5) and (6). Since Love's results are stated for $m=1$, we shall also take $m=1$ in the remainder of the paper. Corresponding results for general $m$ are easily obtained by simple changes of variables.

As usual we shall focus attention on $H_{1}(a, b ; c ; 1)$. We shall have occasion to consider $H_{1}(a, b ; c ; 1) f$ where $f$ is either a classical function or a generalised function. If $f$ is a classical function (for example in $L_{\mu}^{p}$ ) we must assume that $\operatorname{Re} c>0$ and define $H_{1}(a, b ; c ; 1) f$ by $(1.5)$ with $\phi$ replaced by $f$ and $m=1$; that is

$H_{1}(a, b ; c ; 1) f(x)=\int_{0}^{x}(x-t)^{c-1} F^{*}(a, b ; c ; 1-x / t) f(t) d t \quad(x>0)$

with appropriate conditions on $a$ and $b$ to guarantee the existence of the integral. 
If $f$ is a generalised function, we define $H_{1}(a, b ; c ; 1) f$ by Definition 5.3 and use (5.7), with $m=1$.

Working with certain classes of locally integrable classical functions (defined below), Love has to impose considerable restrictions on the parameters and proofs are different for different ranges of the parameters. On the other hand, in our results for generalised functions, the restrictions are less numerous and there is no need to split up proofs into different cases. This is hardly surprising when we consider the powerful tools, such as analytic continuation, which are available for generalised functions but not for locally integrable functions.

In (5), Love gives six solution formulae, valid under appropriate sets of conditions (the sets not being disjoint) for the equation

$$
H_{1}(a, b ; c ; 1) f=g,
$$

where $f, g$ are classical functions satisfying an appropriate local integrability condition. Three of these formulae are

$$
\begin{aligned}
& f(x)=x^{-a} I_{x}^{-b} x^{a} I_{x}^{b-c} g(x) \\
& f(x)=x^{-b} I_{x}^{b-c} x^{c-a} I_{x}^{-b} x^{a+b-c} g(x) \\
& f(x)=x^{-a} \frac{d^{n}}{d x^{n}}\left\{x^{a} H_{2}(-a, n-b ; n-c ; 1) g(x)\right\}
\end{aligned}
$$

(where $n$ is a positive integer, $n-\operatorname{Re} c>0$ and $H_{2}(-a, n-b ; n-c ; 1) g(x)$ is defined using the analogue of $(4.1)$ with $\mathrm{m}=1)$. The other three are obtained by interchanging $a$ and $b$ and using the fact that

(compare (3.8)).

$$
H_{1}(b, a ; c ; 1) f=H_{1}(a, b ; c ; 1) f
$$

As a contrast, we consider (6.2) where $f, g$ are generalised functions and suppose that the conditions of Theorem 5.5 are satisfied with $m=1$, i.e.

$$
\operatorname{Re} \mu-1 / p<\min (\operatorname{Re} a, \operatorname{Re} b, \operatorname{Re} c, \operatorname{Re}(a+b)), g \in F_{p, \mu-c}^{\prime} .
$$

Theorem 5.5 ensures that the right-hand side of (6.3) is the unique solution in $F_{p, \mu}^{\prime}$. However, (6.4) and (6.5) are also valid under the same conditions and are merely alternative ways of writing (6.3). Indeed, if we apply Theorem 5.10 (i) with $\alpha, \beta, \gamma, \mu$ and $f$ replaced by $c-a,-b, a+b-c, \mu-c$ and $g$ (which is permissible under the given conditions)

$$
\begin{aligned}
& x^{-b} I_{x}^{b-c} x^{c-a} I_{x}^{-b} x^{a+b-c} g \\
& =x^{-b} I_{x}^{b-c} I_{x}^{-a-b+c} x^{b} I_{x}^{a-c} g \\
& =x^{-b} I_{x}^{-a} x^{b} I_{x}^{a-c} g
\end{aligned}
$$$$
\text { using (3.31) of (9); }
$$

we have (6.3) with $a$ and $b$ interchanged. As regards (6.5), suppose $n$ is a nonnegative integer; then we may apply Theorem 5.7 with $a, b, c, \mu$ and $f$ replaced 
by $-a, n-b, n-c, \mu-c$ and $g$ to deduce that

$$
\begin{aligned}
x^{-a} & \frac{d^{n}}{d x^{n}}\left\{x^{a} H_{2}(-a, n-b ; n-c ; 1) g\right. \\
& =x^{-a} \frac{d^{n}}{d x^{n}} x^{a} x^{-a} I_{x}^{n-b} x^{a} I_{x}^{b-c} g \\
& =x^{-a} I_{x}^{-n} I_{x}^{n-b} x^{a} I_{x}^{b-c} g \\
& =x^{-a} I_{x}^{-b} x^{a} I_{x}^{b-c} g
\end{aligned}
$$

and we have (6.3) again.

We now indicate how our results involving generalised functions can be $\mathrm{u}$ sed to obtain results concerning classical solutions of (6.2); thus given $g(x)$ d efined for $x \in(0, \infty)$ we try to find $f(x)$ such that

$$
\int_{0}^{x}(x-t)^{c-1} F^{*}(a, b ; c ; 1-x / t) f(t) d t=g(x),
$$

where equality is to hold almost everywhere on $(0, \infty)$. (Here $\operatorname{Re} c>0$ as indicated earlier.) We shall identify functions differing only on a set of measure zero on $(0, \infty)$. In (5), Love discusses (6.6) relative to spaces $Q_{r}$. (We use $r$ rather than $q$ to avoid confusion with $1 / p+1 / q=1$.) By definition, for each real $r$,

$$
Q_{r}=\left\{f: x^{r} f(x) \in L^{1}(0, X) \text { for each } X \in(0, \infty)\right\} \text {. }
$$

We shall discuss (6.6) relative to the spaces $L_{\mu}^{p}$ (see Definition 5.1). Given $f \in L_{\mu}^{p}$, we again denote by $\tau f$ the element of $F_{q,-\mu}^{\prime}$ defined by (5.3). We can regard $f \rightarrow \tau f$ as an imbedding of $L_{\mu}^{p}$ into $F_{q,-\mu}^{\prime}$. We shall use $\tau$ to denote any such imbedding and will not show the dependence on $p, \mu$ explicitly; this should not cause confusion.

It is natural to try to compare the spaces $Q_{r}$ and $L_{\mu}^{p}$. Since local integrability does not imply integrability over $(0, \infty)$, there is no hope of any inclusion of the form $Q_{r} \subset L_{\mu}^{p}$. However, in the other direction we have

Lemma 6.1. $L_{\mu}^{p} \subset Q_{r}$ provided $-\operatorname{Re} \mu-1 / q<r$.

Proof. Let $f \in L_{\mu}^{p}$ so that $\int_{0}^{x}\left|x^{-\mu} f(x)\right|^{p} d x<\infty$ for any $X \in(0, \infty)$. Then, by Hölder's inequality, if $1<p<\infty$,

$$
\begin{aligned}
\int_{0}^{x}\left|x^{r} f(x)\right| d x=\int_{0}^{x} x^{r+R e \mu} \mid & x^{-\mu} f(x) \mid d x \\
& \leqq\left\{\int_{0}^{x} x^{(r+R e \mu) q} d x\right\}^{1 / q}\left\{\int_{0}^{x}\left|x^{-\mu} f(x)\right|^{p} d x\right\}^{1 / p}<\infty
\end{aligned}
$$

since $(\mathrm{r}+\operatorname{Re} \mu) q>-1$ by hypothesis. The proof is similar if $p=1$ or $\infty$. The result follows. 
We know from Lemma 5.2 that if (6.6) is to have a solution $f$ in $L_{\mu}^{p}, g$ must belong to $L_{\mu+c}^{p}$. We could regard this as a very rough analogue of Theorem 7 , p. 185 of (5); however, our condition on $g$ is an integrability condition whereas Love's condition, namely $I_{x}^{-c} g \in Q_{r}$, is more a differentiability condition. We can prove theorems corresponding to other results in (5). For example, compare (5, Corollary 1, p. 179) and

Theorem 6.2. If $g \in L_{\mu+c}^{p}$ where

$$
-\operatorname{Re} \mu-1 / q<\min (\operatorname{Re} a, \operatorname{Re} b, \operatorname{Re} c, \operatorname{Re}(a+b)) \text { and } \operatorname{Re} c>0,
$$

(6.6) has at most one solution $f \in L_{\mu}^{p}$.

Proof. It is instructive to give two proofs.

(i) Choose $r$ such that $-\operatorname{Re} \mu-1 / q<r<\min (\operatorname{Re} a, \operatorname{Re} b, \operatorname{Re} c, \operatorname{Re}(a+b))$. Then also $-\operatorname{Re}(\mu+c)-1 / q<r<\min (\operatorname{Re} a, \operatorname{Re} b, \operatorname{Re} c, \operatorname{Re}(a+b))$ since $\operatorname{Re} c>0$. Thus $g \in L_{\mu+c}^{p} \Rightarrow g \in Q_{r}$ by Lemma 6.1. By Corollary 1 on p. 179 of (5), (6.6) has at most one solution $f \in Q_{r}$. However, under the given conditions, $L_{\mu}^{p} \subset Q_{r}$ by Lemma 6.1 again; the result follows.

(ii) $g$ generates a regular functional $\tau g \in F_{q,-\mu-c}^{\prime}$ via (5.3). Thus applying $\tau$ to (6.6) gives

$$
\tau H_{1}(a, b ; c ; 1) f=\tau g
$$

in $F_{q,-\mu-c}^{\prime}$. Also any solution $f$ in $L_{\mu}^{p}$ of (6.6) generates $\tau f \in F_{q,-\mu}^{\prime}$ and under the given conditions, (5.5) is valid and gives

$$
\tau H_{1}(a, b ; c ; 1) f=H_{1}(a, b ; c ; 1) \tau f .
$$

On the left-hand side $H_{1}(a, b ; c ; 1)$ is defined via $(6.6)$ while on the right-hand side we use Definition 5.3. From (6.8) and (6.9) we seek $f \in L_{\mu}^{p}$ satisfying

$$
H_{1}(a, b ; c ; 1) \tau=\tau g .
$$

Now, under the given conditions, we may apply Theorem 5.5 with $p, \mu$ replaced by $q$ and $-\mu$ to deduce that the equation

$$
H_{1}(a, b ; c ; 1) h=\tau g
$$

has a unique solution $h \in F_{q,-\mu}^{\prime}$ given by

$$
h=x^{-a} I_{x}^{-b} x^{a} I_{x}^{b-c} \tau g .
$$

Thus (6.6) will have either no solution in $L_{\mu}^{p}$ or exactly one solution in $L_{\mu}^{p}$ (since we are identifying functions which differ only on a set of measure zero), depending on whether there exists $f \in L_{\mu}^{P}$ such that $h=\tau f$, that is, depending on whether $h$, as given by (6.12), is a regular functional.

We might call $h$, as given by (6.12), a generalised solution of (6.6). The second proof of Theorem 6.2 has therefore established 
Corollary 6.3. If $g \in L_{\mu+c}^{p}$ with

$-\operatorname{Re} \mu-1 / q<\min (\operatorname{Re} a, \operatorname{Re} b, \operatorname{Re} c, \operatorname{Re}(a+b))$ and $\operatorname{Re} c>0$,

(6.6) has a unique generalised solution in $F_{q,-\mu}^{\prime}$ given by

$$
h=x^{-a} I_{x}^{-b} x^{a} I_{x}^{b-c} \tau g .
$$

If we are interested in classical rather than generalised solutions we must put further restrictions on our parameters and on $g$. We then obtain results analogous to Theorems 8-12 in (5). Selecting just one at random, we shall prove an analogue of Theorem 11. Our proof requires the following lemma.

Lemma 6.4. Let $f \in L_{\mu}^{p}$.

(i) For any complex numbers $\mu$ and $\eta$,

$$
\tau x^{n} f=x^{\eta} \tau f \text {. }
$$

(ii) If $\operatorname{Re} \alpha>0$ and $\operatorname{Re} \mu>-1 / q$,

$$
\tau I_{x}^{\alpha} f=I_{x}^{\alpha} \tau f \text {. }
$$

Proof. We prove (ii); (i) can be proved similarly using (2.13) of (9).

Since $\operatorname{Re} \alpha>0$ and $\operatorname{Re} \mu>-1 / q, I_{x}^{\alpha} f$ is defined by

$$
I_{x}^{\alpha} f(x)=\int_{0}^{x} \frac{(x-t)^{\alpha-1}}{\Gamma(\alpha)} f(t) d t \quad(x>0) .
$$

Under the given conditions we may apply Theorem 2.1 (i) of (4) (with $f(x), \zeta$ replaced by $x^{-\mu} f(x)$ and $\mu$ ) to deduce that $I_{x}^{\alpha} f \in L_{\mu+\alpha}^{p}$ so that $\tau I_{x}^{\alpha} f \in F_{q,-\mu-\alpha}^{\prime}$. On the other hand, $f \in L_{\mu}^{p} \Rightarrow \tau f \in F_{q,-\mu}^{\prime}$ and we may apply Theorem 3.11 (with $p, \mu$ replaced by $q,-\mu$ ) to deduce that $I_{x}^{\alpha} \tau f \in F_{q,-\mu-\alpha}^{\prime}$ also. (Here $I_{x}^{x}$ is interpreted in the sense of (3.29) of (9)). Thus both sides of (6.14) exist and belong to $F_{q,-\mu-\alpha}^{\prime}$. To prove equality let $\phi \in F_{q,-\mu-\alpha}$. Then

$$
\begin{aligned}
\left(I_{x}^{\alpha} \tau f, \phi\right) & =\left(\tau f, K_{x}^{\alpha} \phi\right) \\
& =\int_{0}^{\infty} f(x) K_{x}^{\alpha} \phi(x) d x \\
& =\int_{0}^{\infty} f(x)\left(\int_{x}^{\infty} \frac{(t-x)^{\alpha-1}}{\Gamma(\alpha)} \phi(t) d t\right) d x \\
& =\int_{0}^{\infty} \phi(t) d t \int_{0}^{t} \frac{(t-x)^{\alpha-1}}{\Gamma(\alpha)} f(x) d x \\
& =\int_{0}^{\infty} I_{x}^{\alpha} f(x) \phi(x) d x \\
& \Rightarrow\left(I_{x}^{\alpha} \tau f, \phi\right)=\left(\tau I_{x}^{\alpha} f, \phi\right)
\end{aligned}
$$

The inversion of the order of integration in the fourth line above is justified since the repeated integrals involved are both absolutely convergent by Hölder's inequality. This completes the proof. 
We can now prove

Theorem 6.5. If $\operatorname{Re} b<0, \operatorname{Re} c>0,-\operatorname{Re}(\mu+b)-1 / q<\min (0, \operatorname{Re} a)$ and $I_{x}^{b-c} g \in L_{\mu+b}^{p}$ (that is, there exists $G \in L_{\mu+b}^{p}$ such that $g=I_{x}^{c-b} G$ ), then (6.6) has a unique solution $f \in L_{\mu}^{p}$ given by

$$
f(x)=x^{-a} I_{x}^{-b} x^{a} G(x) .
$$

Proof. Under the given conditions, we certainly have $\operatorname{Re} c>0$ and also $-\operatorname{Re} \mu-1 / q<\min (\operatorname{Re} a, \operatorname{Re} b, \operatorname{Re} c, \operatorname{Re}(a+b))$. Further, since $\operatorname{Re}(c-b)>0$ we may apply Theorem 2.1 (i) of (4) (with $f(x), \zeta$ and $\alpha$ replaced by $x^{-\mu-b} G(x)$, $\mu+b$ and $c-b$ ) to prove that $G \in L_{\mu+b}^{p} \Rightarrow g \in L_{\mu+c}^{p}$. Hence, by Corollary 6.3, (6.6) has a unique generalised solution $h \in F_{q,-\mu}^{\prime}$ given by

$$
\begin{aligned}
h & =x^{-a} I_{x}^{-b} x^{a} I_{x}^{b-c} \tau g \\
& =x^{-a} I_{x}^{-b} x^{a} I_{x}^{b-c} \tau I_{x}^{c-b} G \\
& =x^{-a} I_{x}^{-b} x^{a} I_{x}^{b-c} I_{x}^{c-b} \tau G \\
& =x^{-a} I_{x}^{-b} x^{a} \tau G \\
& =x^{-a} I_{x}^{-b} \tau x^{a} G \\
& =x^{-a} \tau I_{x}^{-b} x^{a} G .
\end{aligned}
$$

Thus, by (6.13) we have

$$
h=\tau x^{-a} I_{x}^{-b} x^{a} G .
$$

The first application of (6.14) above is valid since $\operatorname{Re}(c-b)>0$ and

$$
\operatorname{Re}(\mu+b)>-1 / q
$$

and the second is valid since $\operatorname{Re}(-b)>0$ and $\operatorname{Re}(\mu+b+a)>-1 / q$. From (6.16) we have $h=\tau f$ where

$$
f=x^{-a} I_{x}^{-b} x^{a} G .
$$

By examining the Proof of Theorem 6.2, we see that $f \in L_{\mu}^{p}$ is a solution of (6.6) and since $\tau$ is a 1-1 mapping of $L_{\mu}^{p}$ into $F_{q,-\mu}^{\prime}$, uniqueness of $h$ gives uniqueness of $f$. The result follows.

Here we mention that in (6), Love discusses the operators $H_{3}(a, b ; c ; 1)$ and $H_{4}(a, b ; c ; 1)$ relative to the spaces $R_{r}$; for each real $r$

$$
R_{r}=\left\{f: x^{r} f(x) \in L^{1}(X, \infty) \text { for each } X>0\right\} .
$$

(Compare with (6.7).) Analogously to Lemma 6.1 we find that $L_{\mu}^{p} \subset R_{r}$ provided that $\operatorname{Re} \mu+1 / q<-r$. We can then proceed as above to prove theorems on classical solutions analogous to those in (6). Details are similar to those above and are omitted.

Lemma 5.2 showed that the integrability condition $g \in L_{\mu+c}^{p}$ was necessary for (6.6) to have a solution $f \in L_{\mu}^{p}$. On the other hand, the condition $I_{x}^{b-c} g \in L_{\mu+b}^{p}$ in Theorem 6.5 states that $g$ has a fractional derivative of order $c-b$ (belonging to $L_{\mu+b}^{p}$ ). This is characteristic in the sense that any sufficient condition for a 
classical solution (in $L_{\mu}^{p}$ ) of (6.6) (or the corresponding equations for the other operators) seems to require of $g$ a certain degree of differentiability. For further discussions in this direction we refer the reader to Higgins (3).

In conclusion, we remark that results for special cases such as Jacobi polynomials and Legendre functions can be obtained by the appropriate choices of $a, b, c$ and $m$.

\section{Acknowledgements}

This paper is based on part of the author's Ph.D. thesis written at the University of Edinburgh under the supervision of Professor A. Erdélyi. It is a pleasure to record my thanks to Professor Erdélyi for his help and guidance and to the Carnegie Trust for the Universities of Scotland for the award of a Scholarship.

I am also grateful to the referee for his many useful comments on the original draft of the paper.

\section{REFERENCES}

(1) A. ERdÉLYI, Fractional integrals of generalised functions, J. Austral. Math. Soc. 14 (1972), 30-37.

(2) A. ERdélyi et al., Higher Transcendental Functions, Vol. I (McGraw-Hill, New York, 1953).

(3) T. P. Higgins, A hypergeometric function transform, J. Soc. Indust. Appl.Math. 12 (1964), 601-612.

(4) H. KoBer, On fractional integrals and derivatives, Quart. J. Math. (Oxford) 11 (1940), 193-211.

(5) E. R. Love, Some integral equations involving hypergeometric functions, Proc. Edinburgh Math. Soc. (2) 15 (1967), 169-198.

(6) E. R. Love, Two more hypergeometric integral equations, Proc. Cambridge Philos. Soc. 63 (1967), 1055-1076.

(7) E. R. Love, Two index laws for fractional integrals and derivatives, J. Austral. Math. Soc. 14 (1972), 385-410.

(8) A. C. MCBRIDE, A Theory of Fractional Integration for Generalised Functions with Applications, Ph.D. Thesis, University of Edinburgh (1971).

(9) A. C. McBrIDE, A theory of fractional integration for generalised functions, SIAM J. Math. Anal. (to appear).

(10) A. H. Zemanian, Generalised Integral Transformations (Interscience, New York, 1968).

\section{Department of Mathematics \\ UNIVERSITY OF STRATHCLYDE \\ LIVINGSTONE TOWER \\ 26 RICHMOND STREET \\ Glasgow, G1 1XH}

